\title{
The role of academic health centres to inform evidence-based integrative oncology practice
}

\author{
Jun J. Mao, Robert B. Saper and Margaret A. Chesney
}

David H. Gorski has written an Opinion article challenging the value of integrative oncology (Integrative oncology: really the best of both worlds? Nature Rev. Cancer 14, 692-700 (2014) $)^{1}$. While we agree with some of his critiques of alternative medicine, his synthesis of the literature does not reflect the state of the science and evidence of current integrative oncology research and practice. For example, he called acupuncture "pseudoscience" but failed to appraise recent relevant translational neuroscience literature on the mechanisms of acupuncture. Using functional brain imaging techniques, researchers have found that acupuncture techniques affect neural networks that are critical to pain processing and emotional regulation ${ }^{2,3}$. These findings enhance our understanding of not only the mechanism underlying acupuncture but also the neurophysiological basis of chronic pain. In a recent patient-level metaanalysis of 17,922 individuals with chronic pain, researchers found that acupuncture was more efficacious than sham acupuncture and standard medical care for pain management $t^{4}$. Gorski's interpretation was that acupuncture did not produce minimal clinically important effects and was therefore not useful overall. Our interpretation is that if a patient does not respond to current medical regimens (such as drugs or interventional pain procedures) or prefers to avoid polypharmacy ${ }^{5}$, acupuncture is an acceptable option based on existing evidence.

As physicians and scientists, we aspire to use science to inform the practice of medicine and improve patient outcomes. In order to achieve this, evidence-based medicine requires that we combine research findings, clinical experience, and the patient's unique preferences, values and circumstances ${ }^{6,7}$. Gorski's dismissal of the field of integrative oncology reflects a poor understanding of this emerging field. A large populationbased study found that $60 \%$ of patients with cancer use one or more types of complementary or integrative medicine (typically for wellness, immune enhancement and pain management), which is a higher rate than that seen in the general population ${ }^{8}$. Because of patient preference and demand, the field of integrative oncology, led by academic health centres and cancer centres, has emerged to address the challenges of evidence-based practice. These centres aim to perform rigorous research to understand the mechanisms of complementary and integrative practices, and to evaluate their potential benefit and harm. They also aim to educate patients and trainees, and to develop sustainable models of clinical practice so that selected complementary therapies (based on greater potential benefit than risk) can be practised along with, or "integrated" into, conventional cancer care ${ }^{9}$. A recent exemplar of this effort is the Journal of the National Cancer Institute Monograph issue, entitled The Role of Integrative Oncology in Cancer Survivorship, which was jointly edited by members of the Society for Integrative Oncology and the Consortium of Academic Health Centers for Integrative Medicine and Health ${ }^{10}$.

The field of integrative oncology is young and has both promise and challenges. As this field evolves, adhering to the principles of evidence-based medicine is essential for its proper maturation. This means acknowledging evidence as it accrues rather than selecting only the evidence that supports a particular viewpoint or opinion.

Jun J. Mao is at the Abramson Cancer Center, Perelman School of Medicine, University of Pennsylvania, Philadelphia, Pennsylvania 19104, USA.

Robert B. Saper is at Boston University School of Medicine, 72 East Concord Street, Boston, Massachusetts 02118, USA.

Margaret A. Chesney is at the Osher Center for Integrative Medicine, University of California School of Medicine, 1545 Divisadero Street, San Fransisco, California 94115, USA.

This article was written on behalf of the Consortium of Academic Health Centers for Integrative Medicine.

Correspondence to J.J.M. e-mail:jun.mao@uphs.upenn.edu doi: 10.1038/nrc3822-c3

1. Gorski, D. Integrative oncology: really the best of both worlds? Nature Rev. Cancer 14, 692-700 (2014).

2. Napadow, V. et al. Brain correlates of phasic autonomic response to acupuncture stimulation: an event-related fMRI study. Hum. Brain Mapp. 34, 2592-2606 (2013).

3. Huang, W. et al. Characterizing acupuncture stimuli using brain imaging with FMRI - a systematic review and meta-analysis of the literature. PLOS ONE 7, e32960 (2012).

4. Vickers, A. J. et al. Acupuncture for chronic pain: individual patient data meta-analysis. Arch. Intern. Med. 172, 1444-1453 (2012).

5. Mao, J. J. et al. Acupuncture for hot flashes: decision making by breast cancer survivors. J. Am. Board Fam Med. 25, 323-332 (2012).

6. Montori, V. M., Brito, J. P. \& Murad, M. H. The optimal practice of evidence-based medicine: incorporating patient preferences in practice guidelines. JAMA 310, 2503-2504 (2013).

7. Hoffmann, T. C., Montori, V. M. \& Del Mar, C. The connection between evidence-based medicine and shared decision making. JAMA 312, 1295-1296 (2014).

8. Mao, J. J., Palmer, C. S., Healy, K. E., Desai, K. \& Amsterdam, J. Complementary and alternative medicine use among cancer survivors: a population-based study. J. Cancer Surviv. 5, 8-17 (2011).

9. Kligler, B. \& Chesney, M. Academic health centers and the growth of integrative medicine. J. Natl Cancer Inst. Monogr. 50, 292-293 (2014).

10. Mao, J. J. \& Cohen, L. Advancing the science of integrative oncology to inform patient-centered care for cancer survivors. J. Natl Cancer Inst. Monogr. 50, 283-284 (2014)

Competing interests statement

The authors declare no competing interests. 